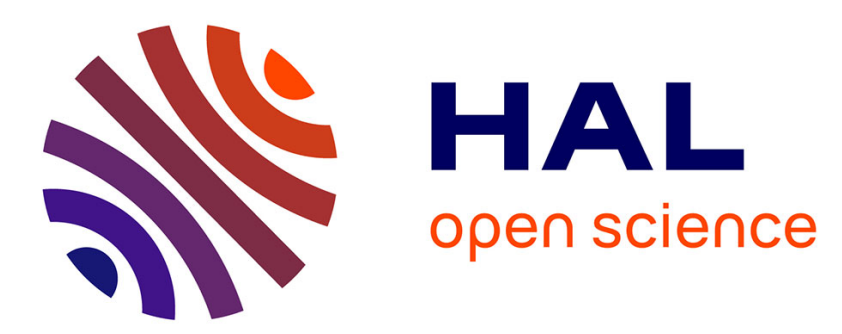

\title{
On the number of clusters and the fuzziness index for unsupervised FCA application to BOLD fMRI time series
}

\author{
Jalal M. Fadili, Su Ruan, Daniel Bloyet, Bernard Mazoyer
}

\section{To cite this version:}

Jalal M. Fadili, Su Ruan, Daniel Bloyet, Bernard Mazoyer. On the number of clusters and the fuzziness index for unsupervised FCA application to BOLD fMRI time series. Medical Image Analysis, 2001, 5 (1), pp.55-67. 10.1016/S1361-8415(00)00035-9 . hal-01121190

\author{
HAL Id: hal-01121190 \\ https://hal.science/hal-01121190
}

Submitted on 27 Feb 2015

HAL is a multi-disciplinary open access archive for the deposit and dissemination of scientific research documents, whether they are published or not. The documents may come from teaching and research institutions in France or abroad, or from public or private research centers.
L'archive ouverte pluridisciplinaire HAL, est destinée au dépôt et à la diffusion de documents scientifiques de niveau recherche, publiés ou non, émanant des établissements d'enseignement et de recherche français ou étrangers, des laboratoires publics ou privés. 


\title{
On the number of clusters and the fuzziness index for unsupervised FCA application to BOLD fMRI time series
}

\author{
M.J. Fadili ${ }^{\mathrm{a}, \mathrm{b}, \mathrm{d}, *}$, S. Ruan ${ }^{\mathrm{a}}$, D. Bloyet ${ }^{\mathrm{a}}$, B. Mazoyer ${ }^{\mathrm{b}, \mathrm{c}}$ \\ ${ }^{\mathrm{a}}$ GREYC-ISMRA UPRESA 6072, 6 Bd Maréchal Juin, 14050 Caen, France \\ ${ }^{\mathrm{b}}$ GIN UPRES EA 2127 \& LRC CEA n ${ }^{\circ}$ 13V, Cyceron, Bd Bécquerel, 14074 Caen, France \\ ${ }^{\mathrm{c}}$ Unité d'IRM, CHU Côte de Nacre, Caen, France \\ ${ }^{\mathrm{d}}$ Department of Experimental Psychology, University of Cambridge, Downing Street, Cambridge CB2 3EB, UK
}

\begin{abstract}
The aim of this paper is to present an exploratory data-driven strategy based on Unsupervised Fuzzy Clustering Analysis (UFCA) and its potential for fMRI data analysis in the temporal domain. The a priori definition of the number of clusters is addressed and solved using heuristics. An original validity criterion is proposed taking into account data geometry and the partition Membership Functions (MFs). From our simulations, this criterion is shown to outperform other indices used in the literature. The influence of the fuzziness index was studied using simulated activation combined with real life noise data acquired from subjects under a resting state. Receiver Operating Characteristics (ROC) methodology is implemented to assess the performance of the proposed UFCA with respect to the fuzziness index. An interval of choice around 2, a value widely used in FCA, is shown to yield the best performance. (C) 2001 Elsevier Science B.V. All rights reserved.
\end{abstract}

Keywords: fMRI; Validity measure; UFCA; Fuzziness index; ROC

\section{Introduction}

Functional MRI is a recent technique for determining the neural correlates of cognitive processes. It can be sensitised to changes of physiological parameters $(\mathrm{CBF}$, CBV, blood oxygenation) during cognitive tasks (Ogawa et al., 1991; Kwong et al., 1992). The most convenient method uses blood as an endogenous contrast agent allowing non-invasive examination of focal signal intensity changes resulting from hemodynamics. The activationinduced increase in blood oxygenation decreases intravascular deoxyhemoglobin and, therefore, decreases susceptibility-induced intravoxel dephasing. Therefore, spin coherence increases, resulting in a relative signal enhancement (Thurlborn, 1982; Ogawa et al., 1991; Ban-

\footnotetext{
*Corresponding author. Tel.: +33-23-145-2920; fax: +33-23-1452698.

E-mail address: jalal.fadili@greyc.ismra.fr (M.J. Fadili).
}

dettini et al., 1992), an effect known as the BOLD contrast. Brain activation is therefore observed as a weak localized signal enhancement in time series images obtained using sequences sensitive to small changes in $T_{2}^{*}$ and $T_{2}$. Many difficulties must be addressed when processing fMRI data such as the weakness of the signal enhancement and the multiple sources of artifacts. These limitations are all the more strict for post-processing strategies since the size of population of the analysed voxels is large.

In order to extract functional information and detect activated regions using fMRI, the most widely adopted procedures are generally based on signal detection theory and are strongly paradigm dependent (Bandettini et al., 1993; Friston et al., 1994; Bullmore and Brammer, 1996; Lange, 1996; Ruan et al., 1996; Kuppussumy et al., 1997). In contrast with these approaches, other authors have applied different methods such as rotated principal component analysis (PCA) and fuzzy clustering analysis (FCA) which have the benefit of being exploratory and model-free 
(Scarth and McIntyre, 1995; Backfrieder, 1996). These techniques have the capability of separating different types of responses (e.g. BOLD from inflow effects), without any knowledge and hypothesis about the paradigm or the hemodynamic response function. FCA is a potent way to investigate fMRI data that has been used successfully, but it also presents several drawbacks. Indeed, the a priori definition of the number of clusters has not been discussed in previous works (Scarth and McIntyre, 1995; Baugmarther et al., 1998; Coutte et al., 1999) and remains an open problem called the 'cluster validity' problem. In addition, the data size remains an important issue since the activated regions represent a small proportion of the brain and can be embedded in the large amount of voxels that are not activated. This is the so-called ill-balanced data problem in the classification literature (Bezdek, 1981, Coutte et al., 1999).

In this paper, the fuzzy c-means (FCM) algorithm is briefly presented and adapted to the fMRI case. The clustering is applied to the data in the temporal domain. The cluster validity problem is then addressed and solved using heuristics (Fadili et al., 1998). An original cluster validity measure is introduced and validated using simulations. The effect of the fuzziness index choice on the detection power is studied using simulations with real fMRI noise (data acquired from subjects under rest condition). These effects are quantified using the area under the ROC as a single index of merit.

The format of the paper is as follows. In Section 2.1, the algorithm is presented in detail. Then, the influence of the Fuzzy Clustering Analysis parameters (e.g. number of a priori clusters, fuzziness index) is studied and discussed in order to optimise the proposed strategy. The emphasis is put on the potential of the method for use in fMRI time series analysis.

\section{Algorithms}

\subsection{The UFCA strategy}

\subsubsection{Overview of the fuzzy c-means (FCM) algorithm}

Fuzzy cluster analysis (Zadeh, 1977) presents an alternative to hard clustering. It attempts to find a partition of a data set $X$ of $n$ feature vectors $\left(\underline{x}_{1}, \underline{x}_{2}, \underline{x}_{3}, \ldots \underline{x}_{n}\right)$, by producing, for a pre-selected number of clusters $c, c$ vectors in a feature space $\mathbb{R}^{p}$, called cluster centroids or prototypes. They represent points around which the data are concentrated. The FCA also produces, for each datum, a membership vector $\underline{u}_{k}$ whose components are real numbers between 0 and 1 , which measures the similarity of the point to each cluster centroid and indicates how well the point is classified. The FCM (Bezdek, 1981) attempts to find a fuzzy partition of the data set by minimising the following within group least-squares error objective func- tion with respect to fuzzy memberships $u_{i k}$ and centroids $\underline{v}_{i}$ :

$J_{m}(X, U, V)=\sum_{i=1}^{c} \sum_{k=1}^{n} u_{i k}^{m} d^{2}\left(\underline{x_{k}} ; \underline{v_{i}}\right)$.

Where $m>1$ is the fuzziness index used to 'tune out' the noise in the data, $n$ is the number of feature vectors $\underline{x}_{k} \in \mathbb{R}^{p}$ (e.g. number of brain voxels in the context of fMRI), $c \geqslant 2$ is the number of clusters in the set and $d\left(\underline{x}_{k}\right.$; $\underline{v}_{i}$ ) is the similarity measure between a datum (voxel time course) and a centroid. The measure $d$ is any metric induced by an inner product on $\mathbb{R}^{p}$, where $p$ is the dimension of the replication space (e.g. number of time series volumes in a fMRI experiment). Minimisation of $J_{m}$ under the following constraints:

(1) $0 \leqslant u_{i k} \leqslant 1 \quad \forall i, k$,

(2) $0<\sum_{k=1}^{n} u_{i k} \leqslant n \quad \forall i$,

(3) $\sum_{i=1}^{c} u_{i k}=1 \quad \forall k$ (i.e. no empty cluster),

yields an iterative minimisation pseudo-algorithm well known as the FCM algorithm (Bezdek, 1981). The components $v_{i l}$ of each centroid $\underline{v}_{i}$ and the membership degrees $u_{i k}$ are updated according to the expressions

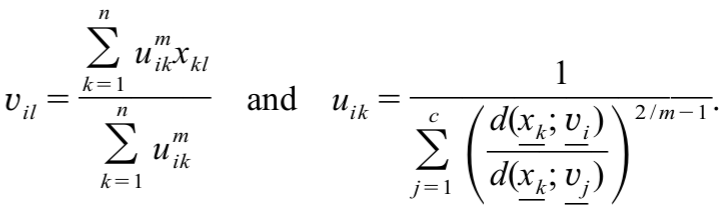

As suggested by (Bezdek, 1981), the membership matrix $U(c, n)$ is initialised randomly or by defining $U^{(0)}(c, n)$ as follows:

$U^{(0)}=\left(1-\frac{\sqrt{2}}{2}\right) U_{u}+\frac{\sqrt{2}}{2} U_{r}$.

Where $U_{u}=[1 / c]$ and $U_{r}$ is a random hard partition of data.

In our context and in order to optimise speed of convergence, the hyperbolic correlation measure was used (Golay et al., 1998). The convergence of the FCM algorithm has been proved in a previous work (Fadili, 1999). Its expression is the following:

$d=d\left(\underline{x_{k}} ; \underline{v_{i}}\right)=\left[\frac{\left(1-c c_{i k}\right)}{\left(1+c c_{i k}\right)}\right]^{\beta}$.

Where $\beta$ is a decreasing speed exponent weight and $c c_{i k}$ is the correlation coefficient between the voxel time course $k$ and the prototype $i$. It should be noted that the action of the index $\beta$ is reflected in the fuzziness degree. As this is controlled using the fuzziness index $m, \beta$ will be held to 1 .

The a priori number of clusters is an important issue for designing a partitional classifier. It is difficult to determine 
the optimal number of clusters. Proposed solutions to this open problem usually rely on validity measures. The cluster validity criterion is a function which assigns to the output of any clustering algorithm a value which is intended to measure the quality of the clustering. By evaluation of this function for various choices of $c$ and $m$, one hopes to be able to determine the $c-m$ values for which the corresponding clustering best identifies the substructures in the data. The valid optimal partition is usually identified as the first local optimum of the validity measure (Bezdek, 1981; Windham, 1982). This is the so-called Unsupervised Fuzzy Clustering algorithm (UFCA). The implementation strategy of the UFCA strategy is summarised in the following pseudo-algorithm $A_{2}$ :

1. Initialise $c=c^{*}=2$, let $U^{*}(c, n)$ and $V^{*}(c, p)$ be the membership and centroids matrices, choose $m$ and a validity measure VF.

2. Apply the FCM algorithm to solve $U$ and $V$ and compute VF value.

3. If optimality of $\mathrm{VF}$ is reached, then $c^{*} \leftarrow c, U^{*} \leftarrow U$, $V^{*} \leftarrow V$ then stop.

4. Else $c \leftarrow c+1$, if $c \geqslant c_{\max }$ then stop; else go to 2 .

Where $c_{\max }=\sqrt{n}$ or $n / 3$ as proposed by many authors (Zahid et al., 1999b).

\subsubsection{Cluster validity and the UFCA}

A good clustering results in well-separated and compact clusters (Dunn, 1974). Several validity criteria have been introduced to assess FCA, however it remains difficult to devise a unique measure that takes into account the variability in cluster shape, density and size. Bezdek (1981) designed the partition coefficient function (PC), associated with the FCM algorithm. In the same vein, similar criteria may be found in the literature such as classification entropy, the proportion exponent, the nonfuzziness index or the modified PC (Bezdek, 1981; Baker and Jain, 1981; Windham, 1982). In spite of their simple implementation, they suffer from lack of connection to any geometrical property of the data since they operate only on the membership degrees. Fukuyama and Sugeno (1989) and Xuanlie and Beni (1991) respectively proposed the so-called $C S$ and $S$ criteria as cluster validity indices. They attempt to minimise the fuzzy intraclass variance of clusters (compactness) and maximise their fuzzy interclass variance (separation). The main advantage of these criteria is that they take into account the geometrical properties of the original set. Nevertheless, $S$ has the disadvantage of presenting a monotonic decreasing behaviour when $c$ gets larger and closer to $n$. Several other criteria have been defined using bootstrap resampling (Jain and Moreau, 1987) or cluster hypervolume and density concepts (Gath and Geva, 1989; Gustafson and Kessel, 1979) to solve the cluster validity problem. They do not necessarily produce the same partition of data. More recently, other measures have been described in the literature such as the fuzzy compactness and separation criterion (Pal and Bezdek, 1995 ) or its modified version proposed in (Zahid et al., 1999a). These last indices are very similar to the $C S$ and $S$ criteria.

We propose a criterion that takes advantage of all previous validity indices. The main idea is based on fuzzy compactness and separation notions. This criterion, denoted $S C F$ can be expressed as follows:

$S C F=S C F_{1}+S C F_{2}$.

Where $S C F_{1}$ and $S C F_{2}$ are measures taking into account fuzzy compactness, separation, union and intersection of the clusters provided by the partition.

$S C F_{1}$ is defined as the ratio of the compactness and average separation between clusters,

$$
S C F_{1}=\frac{\frac{\sum_{i=1}^{c} \sum_{k=1}^{n} u_{i k}^{m} d^{2}\left(\underline{x_{k}}, \underline{v_{i}}\right)}{\pi_{i}}}{\frac{\sum_{i=1}^{c} d^{2}\left(\underline{v_{i}}, \underline{\bar{x}}\right)}{c}} ; \quad \pi_{i}=\sum_{k=1}^{n} u_{i k} .
$$

Where $\pi_{i}$ is the cluster $i$ cardinality. Hence, the minimum of $S C F_{1}$ is reached for the most compact clusters in $\mathbb{R}^{p}$ with highest average separation. This first term takes into account the shape and size of data and is similar to the $C S$ criterion.

The $\mathrm{SCF}_{2}$ criterion expresses the fuzzy relationships between clusters in terms of fuzzy union and intersection. An optimal partition is obtained when the union is maximal and intersection (separation) is minimal. Thus, the $\mathrm{SCF}_{2}$ expression is

$$
\begin{aligned}
& S C F_{2}=\frac{F I}{F U}=\frac{\sum_{i=1}^{c-1} \sum_{j=i+1}^{c} F I_{i j}}{F U} ; \\
& F I_{i j}=\frac{\sum_{k=1}^{n}\left[\min _{i, j}\left(u_{i k}\right)\right]^{2}}{\sum_{k=1}^{n} \min _{i, j}\left(u_{i k}\right)} ; \\
& F U=\frac{\sum_{k=1}^{n}\left[\max _{i \in\{1, \ldots, c\}}\left(u_{i k}\right)\right]^{2}}{\sum_{k=1}^{n} \max _{i \in\{1, \ldots, c\}}\left(u_{i k}\right)} .
\end{aligned}
$$

$F I_{i j}$ is the fuzzy intersection between clusters $i$ and $j$ and $F U$ is the fuzzy union of all clusters of the partition, both bounded to $[0,1]$. To understand Eq. (8), we shall consider the simple case of $c=2$ without any loss of generality. The behavior of $F U$ and $F I$ is illustrated in Fig. 1, for the simple case of two populations drawn from a normal distribution. The FI, FU and their ratio (the Fuzzy Overlap) are plotted as a function of the difference between the two populations' means normalized to their 


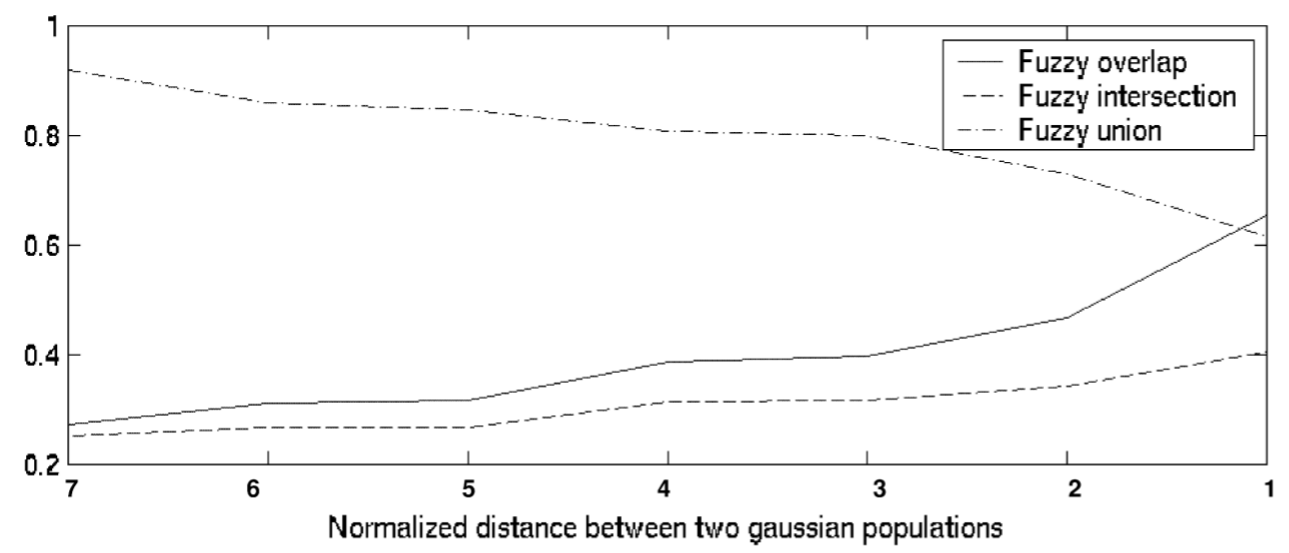

Fig. 1. The behavior of $F U$ and FI for the simple case of two populations drawn from a normal distribution. The $F I, F U$ and their ratio (the Fuzzy Overlap) are plotted as a function of the difference between the two populations' means normalized to their standard deviations.

standard deviations. When the two compared clusters are well separated, the numerator FI tends towards zero while the denominator FU tends to 1 . When the two clusters are likely to overlap, comparable membership degree values are found at the same voxel for the two clusters (the membership function is uniform and tends to $1 / c$ for highly overlapping clusters). Hence, the numerator and the denominator tend to be equal and $S C F_{2}$ approaches 1 .

Finally, minimising the $S C F$ validity measure as described in the algorithm $A_{2}$ provides a valid optimal partition.

\subsubsection{The fuzziness index}

The fuzziness index, also called the fuzziness exponent weight in the fuzzy logic literature, is another important factor in the FCM algorithm. Usually, this parameter is selected according to the problem under consideration. When $m \rightarrow 1$, the fuzzy $c$-means partition tends towards the classical $k$-means hard partition, i.e. $u_{i k} 0$ or 1 . Conversely, when $m \rightarrow \infty$ all clusters tend to the data centroid, i.e. all $u_{i k}$ 's tend to $1 / c$. That is, the higher $m$ is, the fuzzier the partition, and the smaller $m$ is, the crisper the partition. Currently, there is no theoretical basis for an optimal choice for the value of $m$. Furthermore, the $C S$ and $S$ indices become unpredictable for very low and high values of the fuzzy exponent weight $m$. Indeed, these validity measures could be strongly influenced by $m$ since they utilise the provided centroids and membership degrees to calculate the compactness and separation of the fuzzy partition. We carried out a study to propose an acceptable range for the fuzziness index. It is shown that this range includes the most accepted value $m=2$ when using the FCA.

\section{Experiments}

\subsection{Imaging}

The data were acquired using a single shot EPI sequence on a 1.5T Signa scanner (72 blocks of 26 axial interleaved slices, $T_{R}=5 \mathrm{~s}, T_{E}=60 \mathrm{~ms}, \alpha=90^{\circ}, 64 \times 64$ matrix, voxels of $3.75 \times 3.75 \mathrm{~mm}^{2}$ in the plane and $5 \mathrm{~mm}$ thickness with no gap). For the motor activation study, four healthy right-handed young subjects were scanned. The paradigm began with four task volumes and then consisted of eight task-rest cycles, each cycle containing eight volumes (four under each condition). The task was a self-paced right index finger tapping movement. The instructions to switch from one condition to the other were delivered through a microphone. Task execution (extensions and flexions) was visually controlled. Four resting condition sessions (no task), were also acquired for simulation purposes with the same acquisition parameters as above.

Each session was preceded by four dummy volumes to reach the magnetisation equilibrium. These four volumes were discarded from the analysis resulting in $p=68$ temporal samples.

A $T_{1}$-weighted high-resolution scan was also required for anatomical reference: 26 axial slices, $256 \times 256$ matrix with $0.93752 \times 0.93752 \mathrm{~mm}^{2}$ in-plane resolution and $5 \mathrm{~mm}$ slice thickness covering the same field of view as the EPI scans.

\subsection{Preprocessing}

Each series was corrected for the 'slice-dependentphase-shift' effect that is caused by the interleaved scan mode (first even, then odd slices acquired) before any realignment, using cubic-spline interpolation (Fadili, 1999). This step can be crucial for detection sensitivity (Van de Moortele et al., 1996) since the time shift between two extreme slices is approximately equal to the repetition time $T_{R}$ ( $5 \mathrm{~s}$ in our blocked design experiments) which is of the same order as the hemodynamics (Friston et al., 1994). After correction for time shift effects, all the data were corrected for rigid body motion artifacts with a locally written algorithm using a least-squares objective function and a 6 degrees-of-freedom model ( 3 translations and 3 rotations). All volumes were realigned to the same refer- 
ence volume. The detected movements were less then 0.6 $\mathrm{mm}$ and $0.6^{\circ}$. The greatest motion was observed in the $z$ direction. Due to realignment effects, the first and last slices of each volume were also discarded.

Each voxel time course was corrected from low frequency components (drift) using a least-squares detrending procedure (Bandettini et al., 1993). Another approach would be to apply a high pass filtering on the time course. However, the former technique was preferred because the latter requires a paradigm-dependent cut-off frequency choice, which remains empirical.

\section{Results and discussion}

\subsection{Cluster validity}

\subsubsection{Simulation description}

All the presented measures may not lead to the same partition, e.g. the criteria using only membership degrees are usually too restrictive in the case of overlapping nonspherical clusters. The $C S$ and $S$ criteria are more efficient because of their dependence on data structure and shape. However, $C S$ and $S$ can become unpredictable and sensitive to the fuzziness index. Thus, we carried out simulations in order to study the joint influence of $c$ and $m$ on each of the three validity measures $S C F, C S$ and $S$. Hence, for each number of simulated clusters $c_{\text {init }}$, each criterion is calculated as a function of the number of clusters of the partition and the fuzziness index $m$, where $m$ was varied in the range deduced from Section 4.2.

In this study, we used a synthetic fMRI phantom, with brain-shaped axial slices. This way, the number of simulated clusters was controlled and known a priori (Fig. 2). Different configurations of time course, region size and CNR were simulated. Hence, five areas of different sizes were simulated with time courses similar to real life data. As correlation distance was used, the clusters will be distinguished with respect to their prototype shapes. The activated voxels will be simulated as a boxcar reference function convolved with a Poisson function to simulate hemodynamic delay. The relative signal change will range from $2 \%$ to $3 \%$ with Gaussian noise added to the baseline, which is in agreement with the fMRI signal variations expected in the BOLD contrast at 1.5T (Bandettini et al., 1992). The cluster $F$ is the background-only noise cluster (white Gaussian of $3 \%$, which is in the range of noise standard deviation expected in the grey matter observed in the Echo-Planar Imaging sequence at 1.5T). The nonactivation simulated time courses included typical fMRI structural components (i.e. low frequency sinusoidal and linear trend). The other simulation parameters were as follows: series consisting of 68 blocks each of $64 \times 64$ pixels, simulated $T_{R}=5 \mathrm{~s}$ (activation paradigm frequency $0.025 \mathrm{~Hz}: 4$ rest- 4 active- 4 rest... etc.), $\beta=1$, convergence threshold $\varepsilon=0.001$ and hyperbolic correlation distance.

For each number $c_{\text {init }}$ of simulated clusters (background noise only cluster $+{ }^{\prime} c_{\text {act }} \neq 0$ activation clusters' $+{ }^{\prime} c_{\text {init }}-$ $1-c_{\text {act }}$ other clusters'), the UFCA algorithm is applied with $c \in[2, \sqrt{n}]$ and $m \in] 1,2.2]$. The $S C F, C S$ and $S$ criteria are calculated for each situation. For clarity, curves are shown for $c$ in the interval $[2,10]$.

\subsubsection{Simulation results}

Simulation results are depicted in Fig. 3. Each line corresponds to the number $c_{\text {init }}$ of distinct clusters simulated. Each column corresponds to a validity criterion $(C S$, $S$ and $S C F$ ). For each value of initial clusters $c_{\text {init }}$, the most powerful validity criterion is the one providing a clear minimum for $c=c_{\text {init }}$, whatever the value of the fuzziness index. Indeed, this behaviour is true for the proposed SCF criterion where the index $m$ has almost no influence on the value and position of the minimum of the criterion $S C F$ for all simulated numbers of clusters. As predicted, the $C S$ and $S$ criteria are sensitive to $m$. $C S$ has a monotonic decreasing tendency for $m$ greater than 1.7 when $c_{\text {init }}$ becomes large. However, this criterion is still effective and provides a valid partition when $m$ is judiciously chosen. The $S$ criterion is unpredictable for low

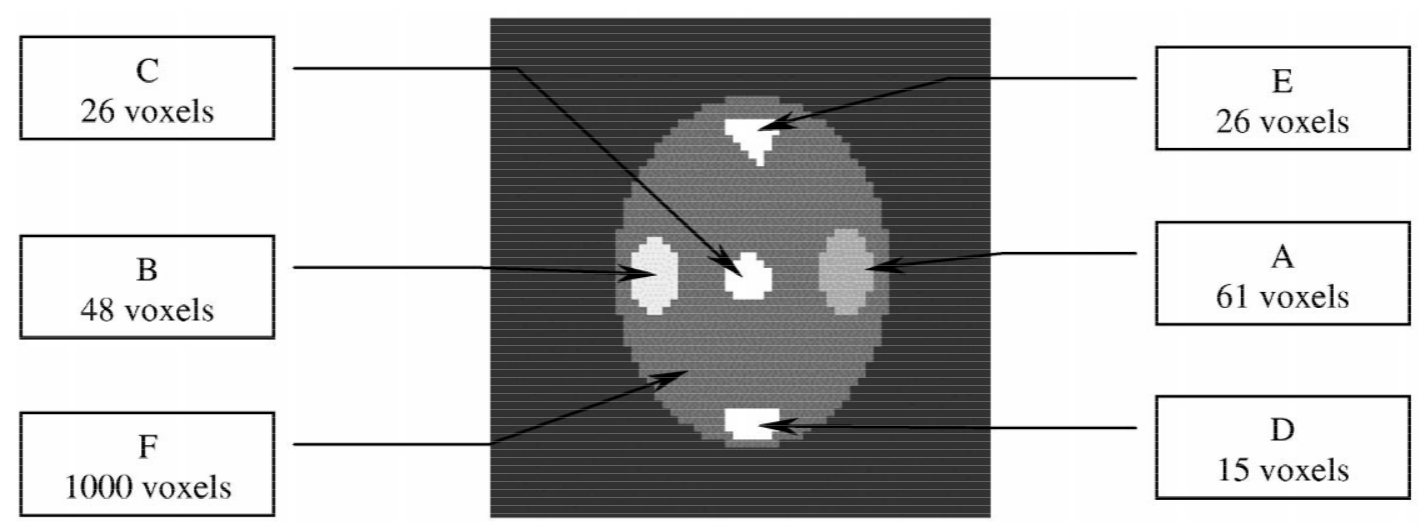

Fig. 2. Synthetic fMRI phantom used for the validation of the UFCA. The clusters are of different shapes, sizes and time profiles. The number of clusters is known and controlled a priori. 


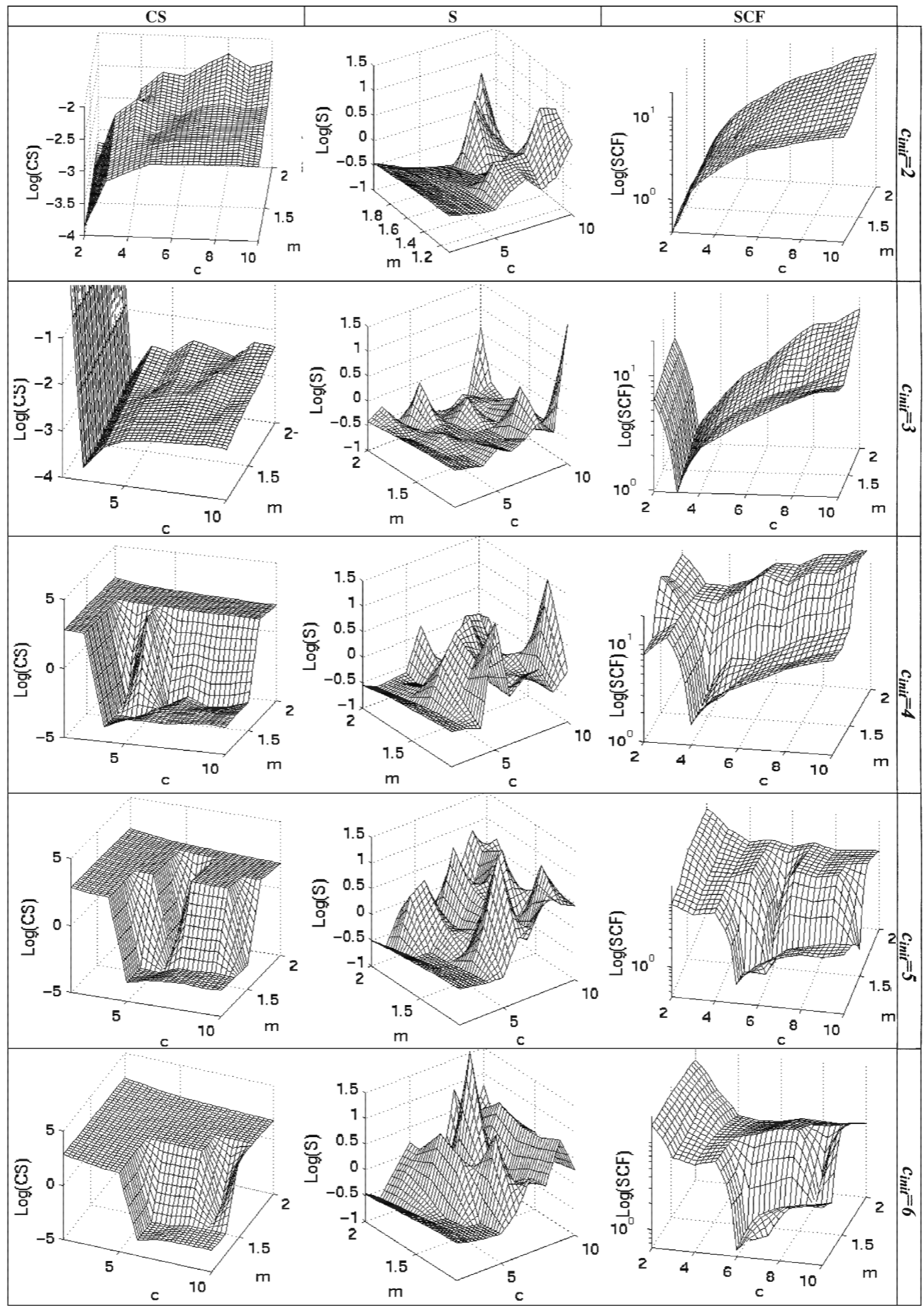

Fig. 3. Simulation results on the synthetic phantom of Fig. 2. Each line corresponds with the number of distinct clusters initially simulated $c_{\text {init }}$, and each column with a validity criterion $(C S, S$ or $S C F)$. A criterion is considered more powerful if its minimum is reached for $c=c_{\text {init }}$ regardless of the value of the fuzziness index $m$. 
values of $c_{\text {init. }}$. This criterion tends to over-partition the data set. The criterion is satisfactory for $c_{\text {init }} \geqslant 4$ and $m$ around 1.5 (compatible with real life fMRI data). Nevertheless, it remains very unstable and several minima could appear (e.g. for $c_{\text {init }}=4$ to 6 ). Thus, the provided partition will be only locally optimal.

These results show the superiority of the proposed criterion SCF and its effectiveness and validity for real-life activation data.

\subsection{Effect of the fuzziness index}

In the context of fMRI, the fuzziness index is very likely to influence detection power. Indeed, choosing an inadequate value for $m$ could lead to the loss of some truly activated voxels (true positives) or, conversely, recover too many truly inactivated voxels (false positives). It is thus necessary to propose a choice ensuring a compromise between specificity and the sensitivity of the method.

\subsubsection{Simulation description}

To assess the effect of the fuzziness index on the FCA algorithm, the simulations were carried-out on the four resting-state data sets. This takes into account realistic properties of the noise without making any assumptions about its distribution or power. Activation patterns with realistic spatial distribution were simulated. Indeed, the spatial extent of the simulated cluster was obtained through real life activated regions corresponding to the motor task (primary motor cortex (PMC), supplementary motor area (SMA) and cerebellum), using a simple correlation coefficient test on the activation series. These regions were then examined visually and manually modified to remove undesirable artifacts. The retained spatial distribution (within the mask of the grey matter) was put in memory. At each memorised voxel, a simulated activation timecourse was injected using an additive model. The response amplitude was adjusted by means of the contrast to noise ratio (CNR) and expressed in terms of relative signal change in percent. For the results presented below, the relative signal change ranged from $0.5 \%$ to $2.5 \%$, which is in the range of functional variations observed in the BOLD contrast at 1.5T (Kwong et al., 1992; Bandettini et al., 1992). Gaussian white noise was also added to the reference boxcar function before convolution, to simulate physiological fluctuations of the subject response. The standard deviation of the added noise ranged from 0 to $2 \%$ of the baseline. The simulated fMRI responses consisted of a boxcar-shaped paradigm convolved with a Poisson function of parameter $\lambda$, which is consistent with previous work (Friston et al., 1994). For the FCA, we used a hyperbolic correlation distance [Eq. (4)].

\subsubsection{Results}

The results are shown in Fig. 4. The MFs are represented for the cluster including activated voxels, as a function of the maximum of the Cross-Correlation Function $(\mathrm{CCF})$ calculated between each voxel profile and the reference activation profile. The simulated activation voxels are depicted with ' + ' and non activated voxels with ' '. Two extreme situations have been simulated: high activation amplitude with low physiological fluctuations (panel (a) where the CNR was set to $2 \%$ and $\sigma_{\text {physio }}=0$ ), and low activation amplitude with high physiological fluctuations (panel (b) where the CNR was $1 \%$ and the simulated physiological noise was equal to $1 \%$ ). In the two cases, the total number of clusters was fixed to 10 .

As a data-driven analysis, it is clear that the UFCA can not stand for a detection step, and additional treatments could be necessary to detect the activation cluster. Indeed, beside the activation-induced cluster, additional clusters could be retained by the method. Many alternatives are then possible to detect the corresponding activation prototype. One could test for significant periodicity via Fourier methods (for periodic paradigms) or for significant correlation to the reference paradigm . . . etc. In our example, a test of periodicity was used.

We can recognise the general behaviour and the influence of the fuzziness index on the partition result (hard to fuzzy for $m$ low to high). Moreover, when $m$ tends to be low and closer to 1, many false positives are identified as activated and similarly, several true positive voxels are lost. Thresholding the membership degree map (MDM) of this cluster in these conditions will result in high loss of true positives and a high false positive fraction in our map. When $m$ is high (typically $m>3$ ), the non-activated and activated voxels are mixed and the defuzzification step becomes harder. In addition, we note that the dispersion of the CCF maximum is larger in the case of non-activated voxels than in the case of the activated voxels. Visual inspection of the $m$ values around 2 suggests that a compromise has been achieved between the two extreme behaviours, which we have just described.

We can assess these results statistically using ROC methods (Sorenson and Wang, 1996) as a partition quality index and the effect on the FCA detection accuracy when varying the index $m$. The area $A_{z}$ under the ROC curves was used as a single index of merit to quantify the detection power (Sorenson and Wang, 1996; Skudlarski et al., 1999). The closer this surface is to unity, the better the detection algorithm. Fig. 5 summarises the previous results by plotting the area under the ROC curves (averaged over the 4 runs) as a function of the fuzziness index for the two sets of simulation parameters. It should be noted that when $m$ becomes larger than 3, the ROC area decreases dramatically for the two sets of simulation parameters. Thus, the range $1.5<m<2.5$ seems to be a good compromise for optimising the performance of the FCA with respect to the exponent weight. A maximum is reached for both parameter sets around $m \approx 2$. In the context of fMRI, this gives a rigorous justification for the popular use of the value 2 for the fuzziness index $m$. 
(a)
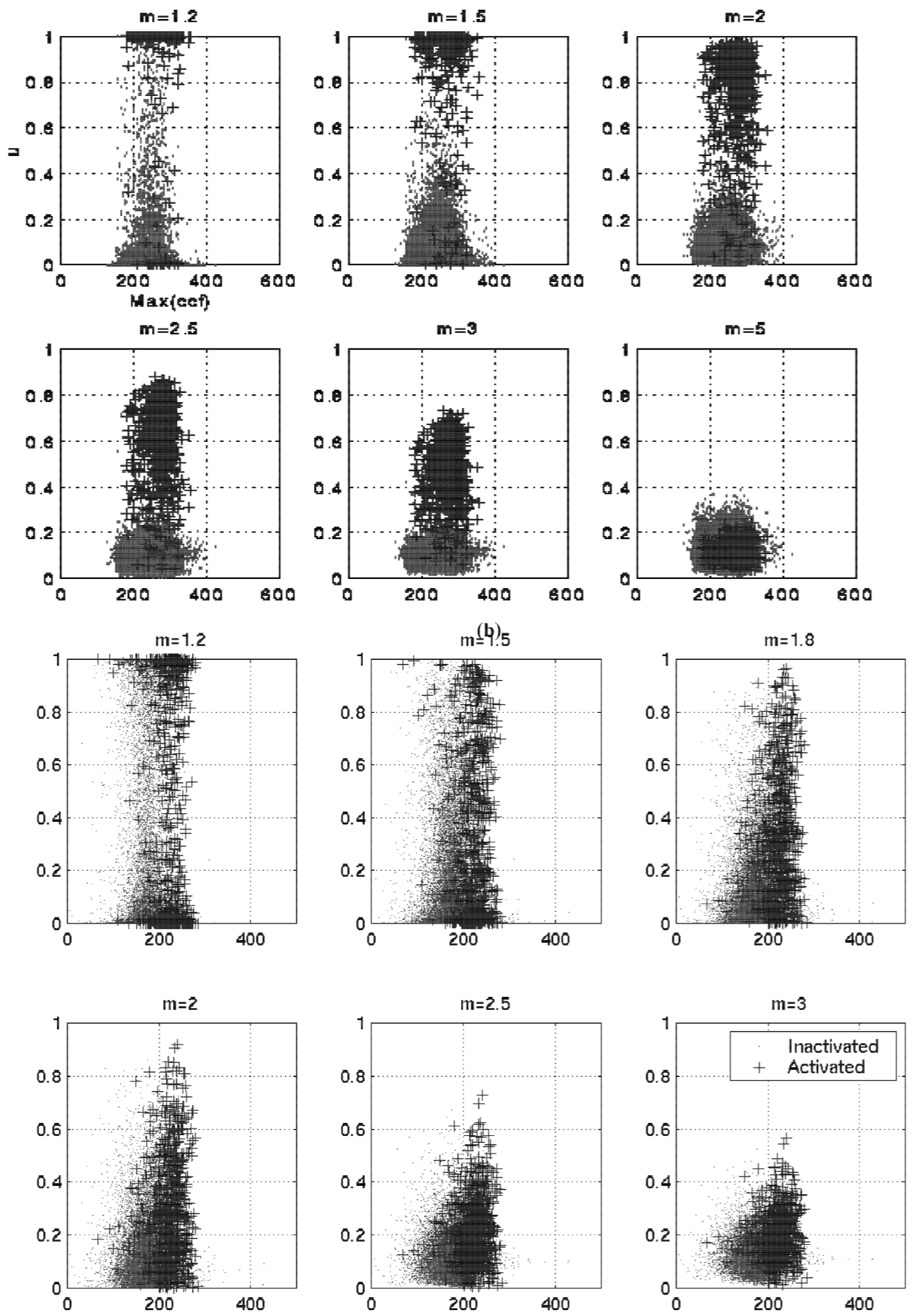

Fig. 4. Influence of the fuzziness index on the fuzzy partition quality for two sets of simulation parameters: $\mathrm{CNR}=2 \%$ (panel a) and $\mathrm{CNR}=1 \%$ physiological noise of $1 \%$ (panel b). A similar tendency of the effect of the fuzziness index is observed for the two cases. Low values of $m$ tend to create hard partition between the non-activated (points) and activated voxels $(+)$. In contrast, the high values are clearly useless because they induce a confusion between activated and non activated voxels. Thus, intermediate values (typically $1.5<m<2.5$ ) seem to be a good compromise for optimizing the performance of the FCA with respect to the exponent weight. 


\section{Area $A_{z}$ versus $m\left(C N R=2 \sigma_{\text {phy }}=0\right)$}

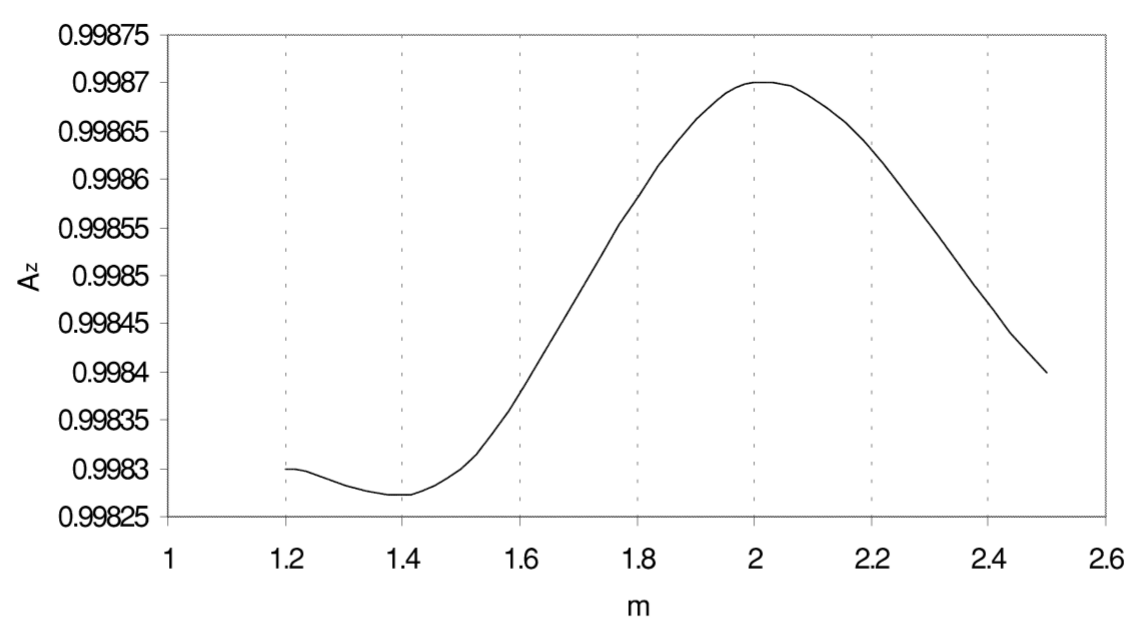

Area $A_{z}$ vs $m\left(C N R=1 \sigma_{\text {phy }}=1 \%\right)$

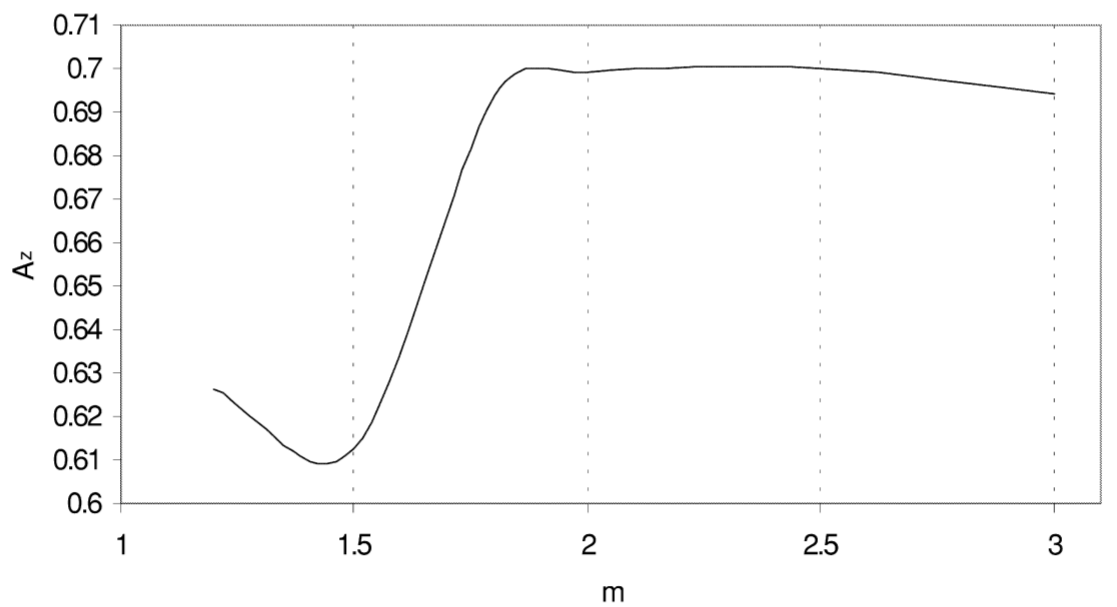

Fig. 5. Statistical assessment, using ROC methods, of the effect of the fuzziness index on the performance of the FCA with real life noise data and simulated activation as in Fig. 4. The performance criterion is the area under the ROC curve. A maximum is reached each time around $m=2$ which is the most common choice for the fuzziness index. Furthermore, the ROC curves for a FPF interval ranging from 0 to 0.01 (which is in the working regime of fMRI) are better for $m \approx 1.8$ to 2 .

\subsection{Application to real-life fMRI activation data}

Our UFCA algorithm was applied to the 4 motor task series described in Section 3.1. The $S C F$ criterion was used as a validity measure for the UFCA and setting $m=2$, $\varepsilon=0.001$. The $S C F$ index indicated a valid optimal partition for $c^{*}=8,10,10$ and 9 clusters for the 4 data sets. As before, the activation cluster was identified using a periodicity test.

\subsubsection{The activation cluster}

The MDMs corresponding to the activation clusters were very reproducible and their mean is depicted in Fig. 6(a). The expected motor areas (PMC, SMA and cerebellum) can be recognised. The fuzzy overlap coefficient FO (Fadili et al., 2000) between pairs of activation MDMs ranged from $76 \%$ to $92 \%$. The retained prototypes are also very similar in shape and signal enhancement (Fig. 6(b)). Indeed, the relative signal change was about $2 \%$ for all cases. The inter-correlation coefficient between the 4 prototypes was also very high (ranging from $c c=0.74$ to $0.9)$. This range of signal variation and the shape of the prototypes are in very good agreement with the realistic simulated activation response used in the simulations. From Fig. 6(b), the shapes of the prototypes suggest that modelling the hemodynamic response may be a hard task. 

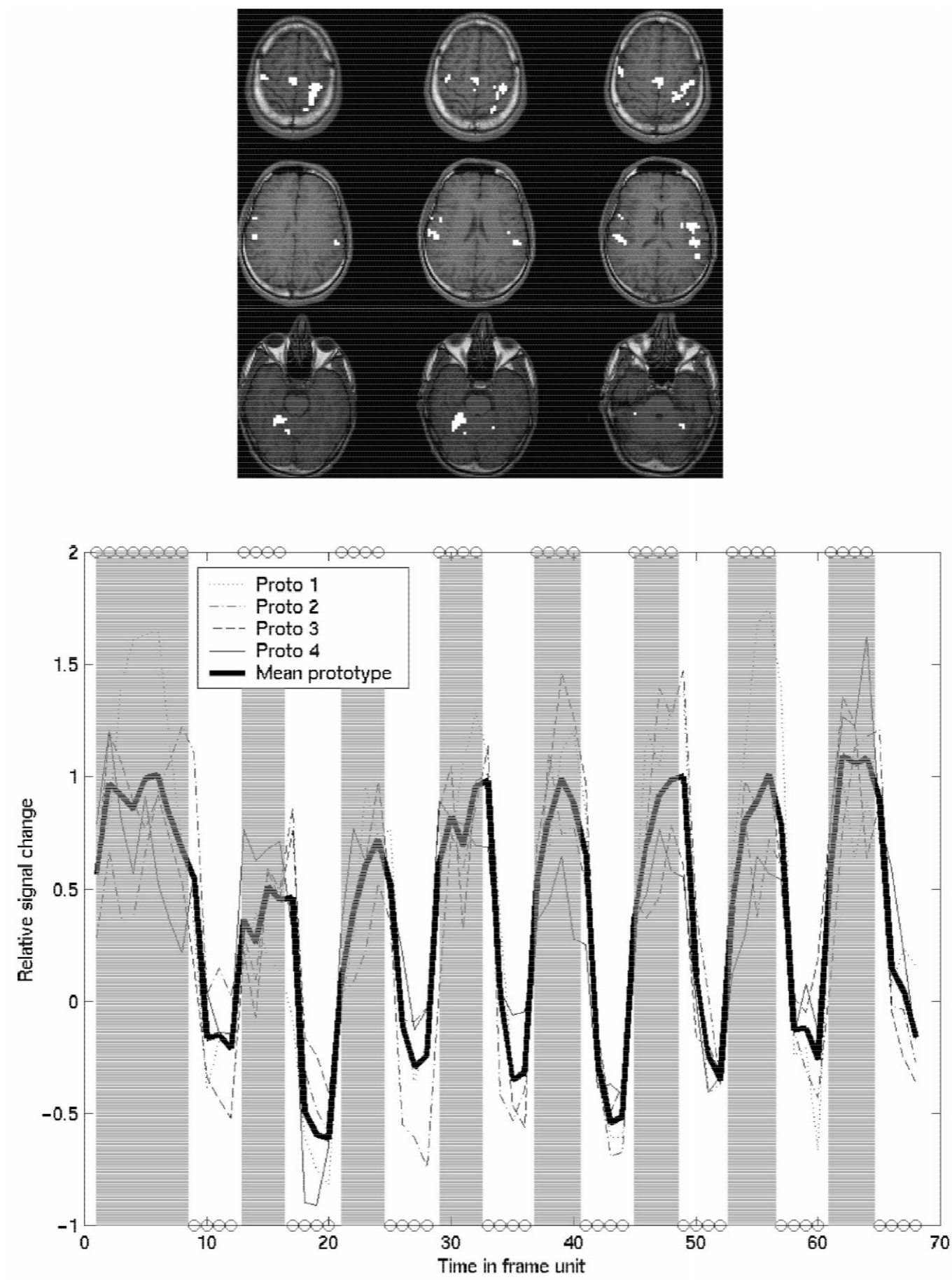

Fig. 6. (a) Spatial (mean MDM thresholded at 0.7) and (b): temporal (prototypes and mean prototype) characteristics of the activation clusters resulting from the UFCA applied to 4 real activation data sets.

This is all the more complex since the response could vary across space, tasks and time (dynamic effect).

FCA has previously been compared to the most widely used detection algorithms (e.g., linear models, correlation techniques) using simulated responses, on realistic noise background (null-hypothesis data), and the ROC methods to statistically assess their differences (Fadili, 1999; Fadili et al., 2000). Here, only the main results are reported and we recommend that the interested reader refer to the references listed above for more detail. The obtained performances were satisfying and similar drawbacks were found for all the approaches with respect to the CNR and the physiological fluctuations. However, the FCA approach was much more stable with respect to the paradigm 
properties such as the number of time points, paradigm period and the conditions' alternation frequency. Similar results on the model-dependent techniques have been reported in (Skudlarski et al., 1999).

\subsubsection{Comments on the other clusters}

The UFCA provides, in addition to the activation cluster, a number of other classes. Most of these are dominated by different sources of noise ("structural» or random components). In the context of fMRI data, it is still a major concern to interpret the huge amount of information provided by the exploratory methods in general (e.g., Principal Components Analysis, Independent Components Analysis, Neural networks) and the FCA in particular. We have introduced a first approach in this direction (Fadili et al., 2000). In this work, the idea was to characterize statistically the obtained task series clusters by using an extra acquisition with no task that uncovers the 'noise' clusters. This step produces some pertinent clusters, in the sense that they are significantly different from those appearing in the noise data set. The reproducibility of the clusters was also assessed using appropriate measures such as the fuzzy overlap coefficient (Fadili, 1999; Fadili et al., 2000).

As the fMRI data are a realization of a complex spatiotemporal process, the noise properties are hard to characterize. Previous studies have empirically shown the $1 / f$ type behavior of the fMRI noise (Aguirre et al., 1997; Zarahn et al., 1997), whose sources have been demonstrated to be instrumental and physiological. Therefore, if the whiteness of the noise is a good approximation at high frequency range, this assumption becomes unrealistic at lower frequencies. From some preliminary studies we have carried out, fractal long-memory processes (e.g., fractional Brownian motion or the associated fractional stationary increment process (Beran, 1994)), seem to be a very good model for the fMRI noise. The fractional Brownian motion is also attractive in the context of fMRI as it is nonstationary by definition (at least in the second order sense which is the point of interest for clustering purposes). Comparisons between simulated realizations of such processes and the "noise" prototypes obtained by the UFCA present a striking resemblance (Fig. 7). The long-range dependence of these noise processes naturally exhibits a slow trend component that is still a major problem when dealing with fMRI data. A number of earlier papers on fMRI (e.g., Bandettini et al., 1993) attempted to propose empirical approaches, such as detrending or high-pass filtering of the time courses, to get rid of trend components, which are badly understood in the literature. This is still the most widely applied technique in the neuroimaging community. However, our belief is that these components are a main feature of the noise itself that could be accounted for by using an appropriate noise model. Our research is now directed towards this approach taking advantage of the orthonormal wavelet transform and its whitening and stationarising properties (Flandrin, 1992; Wornell, 1992).

\section{Conclusion}

This paper has presented a strategy for analysis of fMRI time series using a partitional fuzzy clustering method based on the FCM algorithm. We have described the algorithm, addressed its parameters and limitations. We have suggested some original solutions to the choice of the parameters, and validated these solutions.

The influence of the fuzziness index was studied in detail. Its impact on the partition quality was analysed using the MFs. It was then quantified by using the ROC methodology. In particular, it was shown that the interval in which the performance of the FCA is optimised, includes the value $m=2$. This value has been used by previous authors, but without formal justification or validation.

A solution to the supervision problem (the number of clusters a priori) is proposed, using a heuristic approach. Indeed, the supervision problem constitutes a limitation to the implementation of the FCA for fMRI data analysis and has not been discussed previously by other authors. This led us to develop an algorithm that we have called UFCA. A validity criterion is introduced whose goal is to assess the partition quality. This validity measure was then validated and compared to some well-established criteria resulting from work in the pattern recognition field. The proposed criterion offers high flexibility with the type of distance used (hyperbolic correlation). The simulation study, using regions and time courses of realistic shape, size, amplitude and noise, demonstrated the superiority of our criterion compared to existing criteria. In addition, it was in reasonably good agreement with the $C S$ criterion. The stability of $S C F$ with respect to the index $m$ in the proposed range was also highlighted. In our experience, and for the functional series we have analysed, the optimal number of a priori clusters was generally in a relatively stable interval. If this were generally the case, then this might question the real contribution of the unsupervised approach. However, its interest is indisputable when the data set is different, the distance is different or for a non-experienced user for whom this choice can be a major difficulty. Moreover, solving the supervision problem by choosing a number of clusters that is too high will tend to over-partition the data and consequently to decrease the dynamics of the MDM (Fadili, 1999).

\section{Notation}

fMRI: functional Magnetic Resonance Imaging; FCM: Fuzzy c-means; 

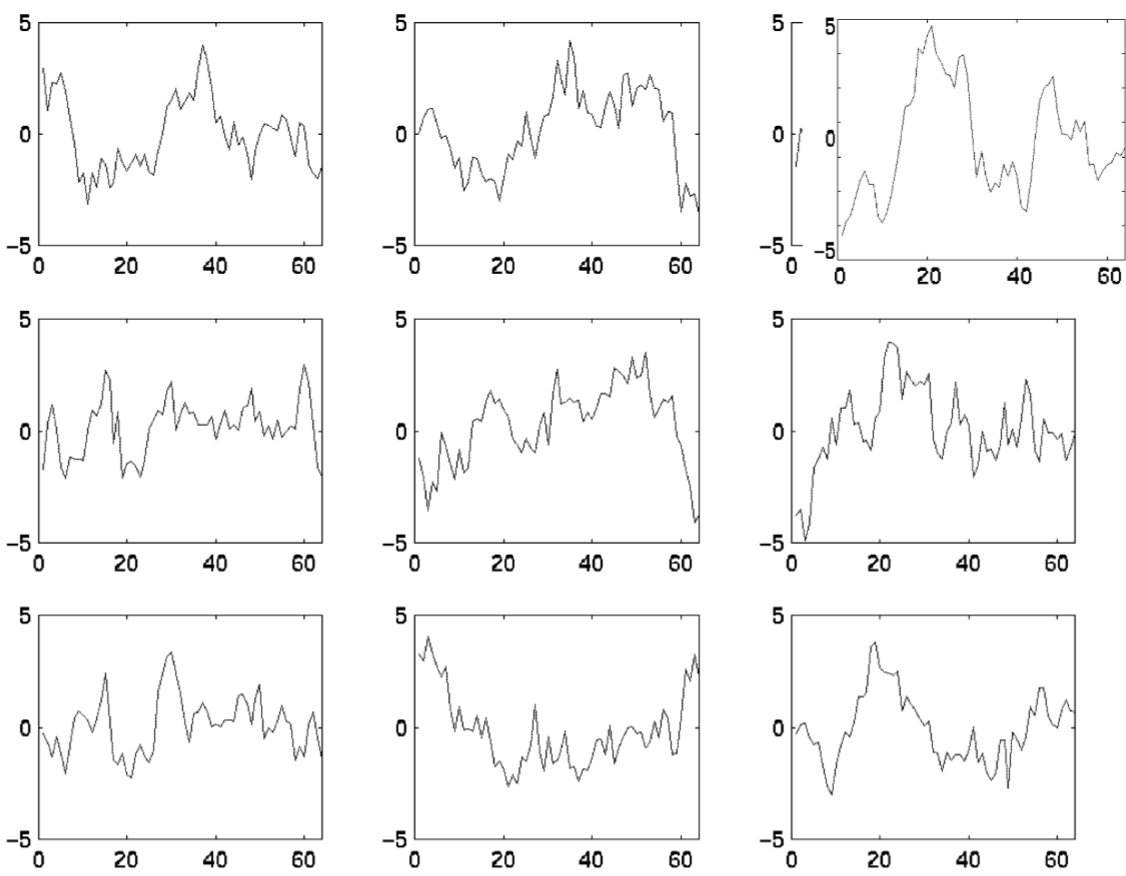

(b)
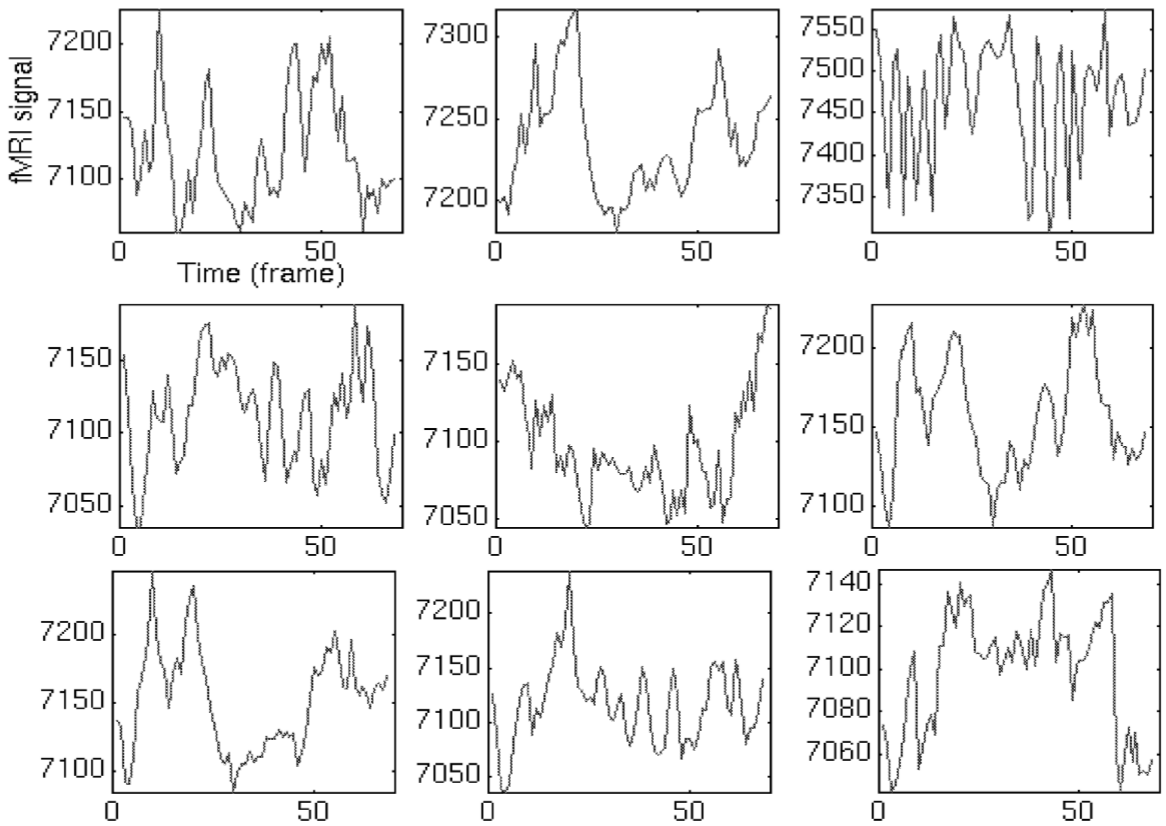

Fig. 7. (a) Examples of fractional Brownian motion process realizations (with different Hurst exponent (Beran, 1994)). (b) Nine non-activation non-detrended prototypes obtained on a typical session from one subject. The 10th prototype corresponded to the motor activation and is displayed in Fig. $6(b)$.

FCA: Fuzzy Clustering Analysis;

UFCA: Unsupervised Fuzzy Clustering Analysis;

VF: Validity Function;

CNR: Contrast-to-Noise Ratio;

MDM: Membership Degree Map;

MF: Membership Function;

ROC: Receiver Operating Characteristics

\section{Acknowledgements}

We would like to thank the anonymous reviewers for very helpful comments and suggestions concerning the first version of this paper. A part of this work is presented in the 6th international conference of Functional Mapping of the Human Brain San Antonio'2000. 


\section{References}

Aguirre, G.K., Zarahn, E., D'Esposito, M., 1997. Empirical analyses of BOLD fMRI statistics: II. Spatially smoothed data collected under null-hypothesis and experimental conditions. NeuroImage 5, 199-212.

Backfrieder, W., 1996. Quantification of intensity variations in functional MR images using rotated principal components. Phys. Med. Biol. 41, $1425-1438$

Baker, B., Jain, A.K., 1981. A clustering performance measure based on fuzzy set decomposition. IEEE Trans. PAMI 3, 66-74.

Bandettini, P.A., Wong, E.C., Hinks, R.S., Tikofsky, R.S., Hyde, J.S., 1992. Time course EPI of human brain function during task activation. Magn. Reson. Med. 25, 390-397.

Bandettini, P.A., Jesmanowiz, A., Wong, E.C., Hyde, J.S., 1993. Processing strategies for time course data sets in functional MRI of the human brain. Magn. Reson. Med. 30, 161-173.

Baugmartner, R., Windischberger, C., Moser, E., 1998. Quantification in functional Magnetic Resonance Imaging. Fuzzy clustering vs. correlation analysis. Magn. Res. Med. 16, 115-125.

Beran, J., 1994. Statistics For Long-Memory Processes. Chapman \& Hall, New York; London.

Bezdek, J.C., 1981. Pattern Recognition With Fuzzy Objective Functions Algorithms. Plenum, New York.

Bullmore, E., Brammer, B., 1996. Statistical methods of estimation and inference for functional MR image analysis. Magn. Res. Med. 35, 261-277.

Coutte, C., Toft, P., Rostrup, E., Nielsen, F.A., Hansen, L., 1999. On clustering fMRI time series. NeuroImage 9, 298-310.

Dunn, J.C., 1974. A fuzzy relative of the ISODATA process and its use in detecting compact well separated clusters. J. Cybern. 3, 32-57.

Fadili, M.J., 1999. Analyse spatio-temporelle des signaux d'activation cérébrale en IRM fonctionnelle. Ph.D. dissertation, Université de Caen.

Fadili, M.J., Ruan, S., Bloyet, D., Mazoyer, B., 1998. Unsupervised fuzzy clustering analysis of fmri series. In: Proc. IEEE EMBS, pp. 696-699.

Fadili, M.J., Ruan, S., Bloyet, D., Mazoyer, B., 2000. A multi-step unsupervised fuzzy clustering analysis of fMRI time series. Human Brain Mapping 10, 160-178.

Flandrin, P., 1992. Wavelet analysis and synthesis of fractional Brownian motion. IEEE Trans. Inf. Theory 38, 910-917.

Friston, K.J., Jezzard, P., Turner, R., 1994. Analysis of functional MRI time series. Human Brain Mapping 1, 153-171.

Fukuyama, Y., Sugeno, M., 1989. A new method for choosing the number of clusters for fuzzy $c$-means method. In: 5th Fuzzy System Symposium, pp. 247-250.

Gath, I., Geva, A.B., 1989. Unsupervised optimal fuzzy clustering. IEEE Trans. PAMI 11, 773-781.

Golay, X., Kollias, S., Stoll, G., Meier, D., Valavanis, A., Boesiger, P., 1998. A new correlation-based fuzzy logic clustering algorithm for fMRI. J. Magn. Res. Med. 40, 249-260.
Gustafson, E., Kessel, W., 1979. Fuzzy clustering with a fuzzy covariance matrix. In: Proc. IEEE CDC, pp. 761-766.

Jain, A.K., Moreau, J.V., 1987. Bootstrap technique in cluster analysis. Pattern Recogn. 20, 547-568.

Kuppussumy, K., Lin, W., Haacke, M., 1997. Statistical assessment of cross-correlation and variance methods and the importance of electrocardiogram gating in fMRI. Magn. Res. Imag. 15, 169-181.

Kwong, K., Belliveau, J. et al., 1992. Dynamic MRI of human brain activity during primary sensory stimulation. Proc. Natl. Acad. Sci. 89, $5675-5679$.

Lange, N., 1996. Statistical approaches to human brain mapping by functional magnetic resonance imaging. Statistics Med. 15, 389-428.

Ogawa, S. et al., 1991. Brain magnetic resonance imaging with contrast dependent on blood oxygenation. Proc. Natl. Acad. Sci. 87, 98689872.

Pal, N.R., Bezdek, J.C., 1995. On cluster validity for the fuzzy $c$-means model. IEEE Trans. Fuzzy Sets and Systems 3, 370-379.

Ruan, S., Jaggi, C., Constans, J., Bloyet, D., 1996. Detection of brain activation from functional MRI data. J. Neuroimag. 6, 207-212.

Scarth, G., McIntyre, M., 1995. Detection of novelty in functional images using fuzzy clustering. In: Proc. ISMRM Nice 1, p. 238.

Skudlarski, P., Constable, R.T., Gore, J.C., 1999. ROC analysis of statistical methods used in functional MRI, individual subjects. NeuroImage 9, 311-329.

Sorenson, J.A., Wang, X., 1996. ROC methods for evaluation of fMRI techniques. Magn. Res. Med. 36, 737-744.

Thurlborn, K.R., 1982. Oxygenation dependence of the transverse relaxation time of water protons on whole blood at high field. Biochim. Biophys. Acta 714, 265-270.

Van de Moortele, P.F., Lobel, E., Paradis, A.L., LeBihan, D., 1996. Brain activation analysis of epi fmri time series needs correction for slicedependent phase shift. In: 2nd International Conference on the Human Brain Mapping, p. 1666.

Windham, M., 1982. Cluster validity for the fuzzy $c$-means clustering algorithm. IEEE Trans. PAMI 4, 357-363.

Wornell, G.W., 1992. Wavelet-based representations for the $1 / f$ family fractal processes. Proc. IEEE 81, 1428-1450.

Xuanlie, L., Beni, G., 1991. A validity measure for fuzzy clustering. IEEE Trans. PAMI 13, 841-847.

Zadeh, L.A., 1977. Fuzzy sets and their application to pattern classification and clustering analysis. In: Rysin, J.V. (Ed.), Classification and Clustering. Academic, New York.

Zahid, N., Aboulala, O., Limouri, M., Essaid, A., 1999a. Unsupervised fuzzy clustering. Pattern Recogn. Lett. 20, 123-129.

Zahid, N., Limouri, M., Essaid, A., 1999b. A new cluster-validity for fuzzy clustering. Pattern Recogn. 32, 1089-1097.

Zarahn, E., Aguire, G.K., D’Esposito, M., 1997. Empirical analyses of BOLD fMRI statistics: I. Spatially unsmoothed data collected under null hypothesis conditions. NeuroImage 5, 179-197. 